\title{
RESEARCH OF RHEOLOGICAL PROPERTIES OF COMPONENTS OF THE DEVELOPED MILK-CONTAINING THERMOSTABLE FILLINGS
}

\author{
Olena Koshel \\ Department of Food Technology ${ }^{1}$ \\ koshelolena85@ukr.net \\ Fedor Pertsevoy \\ Department of Food Technology $y^{1}$ \\ kaf_th@meta.ua \\ Sergei Sabadash \\ Department of engineering technology of food production ${ }^{1}$ \\ s.v.sabadash@ukr.net \\ Mykola Mashkin \\ Department of Milk and Meat Technology $y^{1}$ \\ kalin42@ukr.net \\ Valentyna Mohutova \\ Department of technologies of food production ${ }^{2}$ \\ vf.mogutova@gmail.com \\ Vadym Volokh \\ Department of mechanization of production processes in the agroindustrial complex ${ }^{2}$ \\ volokh69vo@gmail.com \\ ${ }^{1}$ Sumy National Agrarian University \\ 160 G. Kondratyev str., Sumy, Ukraine, 40021 \\ ${ }^{2}$ Luhansk National Agrarian University \\ 68 Slobozhanska str., Starobilsk, Ukraine, 92703
}

\begin{abstract}
The research is devoted to the determination of the properties of the components of the developed milk-containing thermostable fillings and the establishment of the dependence of the effective viscosity on the temperature of the model system. In the work, studies are carried out for a model system containing xanthan gum; a model system containing tara gum; model system containing gelatin; a model system containing xanthan gum and tara gum; a model system containing xanthan gum, tara gum and gelatin; a model system containing xanthan gum, tara gum and sugar; model system containing xanthan gum, tara gum, skimmed milk powder; a model system containing xanthan gum, tara gum and maltodextrin.

In this work, the dependence of the effective viscosity on the temperature of the model system is established. To achieve the aim, the following objectives are set:

- determination of the dependence of the effective viscosity on the temperature of model systems with xanthan gum, tara gum, gelatin, sugar, skimmed milk powder and maltodextrin with different concentrations of these components;

- establishment of the temperature range in which a sharp increase in the effective viscosity of the investigated model systems begins.

An increase in the temperature of the system prevents gelation due to an increase in the intensity of Brownian motion and a decrease through it in the duration of the existence of bonds that arise between macromolecules. At the same time, a decrease in temperature promotes gelation, since this increases the number of contacts between macromolecules, which contributes to an increase in the strength of the so-called spatial network.

In the article, the dependences of the effective viscosity on the temperature of model systems with xanthan gum, tara gum, gelatin, sugar, skimmed milk powder and maltodextrin with different concentrations of these components are obtained. Based on the obtained dependences, the established temperature ranges in which a sharp increase in the effective viscosity of the studied model systems begins.

Keywords: xanthan gum; tara gum; structure formation; gel strength, polysaccharides, viscosity, rheological properties, thermal stability.
\end{abstract}

DOI: $10.21303 / 2504-5695.2020 .001437$ 


\section{Introduction}

One of the technological operations in obtaining the developed fillings is the dispersion of a mixture of components with water at a speed of rotation of the moving parts of the dispersant of $1500 \mathrm{rpm}$. Obviously, the energy consumption for the dispersion process and the load on the moving parts of the dispersant substantially depend on the effective viscosity of the model system to be mixed. In this case, it is possible to influence the efficiency of the dispersion process by varying the rotation frequency of the moving parts of the dispersant and directly by the rheological characteristics of the model systems. The determination of the energy efficiency of the mixing process depending on the frequency of the moving parts of the disperser was not carried out, since this direction of research is limited by the technical characteristics of the selected apparatus.

It should also be noted that the amount of added components is determined by the formulation of the developed filling and affects a number of technological factors of its production, limits the possibility of changing their amount to change the corresponding rheological properties.

However, it is possible to change the effective viscosity of the developed model systems by changing the temperature of the mixture during dispersion.

\section{Literature review and problem statement}

In work [1], the authors carried out research on the technological, physicochemical and rheological properties of gel bases based on hydrocoloids for the further development of gel food products. The objects of research are xanthan gum, guar gum and locust gum, as well as gel bases with different concentrations of these gums. Three methods of studying the technological conditions for the preparation of gel bases are investigated. All experimental gel samples are characterized by a non-Newtonian pseudoplastic flow type. When studying the dependence of the structural viscosity on the shear stress of the samples, it was found that the viscosity of xanthan gels prepared by three methods decreased with an increase in the shear stress. This dependence characterizes xanthan gels as structured dispersed systems. The most suitable is the preparation of gels by the third method, which consisted of swelling the hydrocolloid for 40 minutes in water at room temperature, heating to $60{ }^{\circ} \mathrm{C}$ and cooling. The viscosity of the samples prepared in this way (with the same concentration of gelling agent) is higher and the gel structure was homogeneous.

Optimal concentration of gelling agents to ensure effective structure. The base is $0.5 \%$. These gels have a high strength of structural links and a wide range of structural failure stresses; they also meet the necessary organoleptic properties: they have a more uniform structure, without lumps and do not delaminate during the technological process. In this work, studies of the rheological properties of gel bases based on hydrocolloids have been carried out, but the dependence of the effective viscosity on the temperature of model systems has not been studied, and the regularities of systems at different temperatures of hydration have not been established.

In work [2] the properties of gels of tara gum mixed with cap-Carrageenans or xanthan gum are given. Tara gum is a natural gelling polymer that can form a gel when mixed with cap-Carrageenans and xanthan gum. The synergistic effect of the gel is possible due to intermolecular interactions. In the work, studies of the rheological properties of gel bases based on hydrocolloids are carried out, but the dependence of the effective viscosity on the temperature of model systems was not investigated and the regularities of systems at different temperatures of hydration were not established.

The results of an analytical review of binary combinations in [3], on the basis of which two binary combinations were discovered - "xanthan gum-Konjac gum" and "xanthan gum-tara gum" are presented. It has been found that the use of the combination allows a jelly-like system to be obtained. In the course of experimental studies, the authors confirm the synergistic interaction in the systems "xanthan gum-Konjac gum" and "xanthan gum-tara gum", and also chose a rational ratio of the mixture of xanthan gum-tara gum as 60:40.

\section{The aim and objectives of research}

The aim of the presented research is to establish the dependence of the effective viscosity on the temperature of the model system. To achieve the aim, the following objectives are set: 
- determination of the dependence of the effective viscosity on the temperature of model systems with xanthan gum, tara gum, gelatin, sugar, skimmed milk powder and maltodextrin with different concentrations of these components;

- establishment of the temperature range in which a sharp increase in the effective viscosity of the investigated model systems begins.

Since there is not enough data to solve the above issues, it is necessary to deepen and expand research in determining the effective viscosity of model systems with xanthan gum, tara gum, gelatin, sugar, skimmed milk powder and maltodextrin with different concentrations.

\section{Materials and methods of research}

The studies were carried out on a rotational viscometer. The rheological curves were used to determine the effective viscosity for the shear rate, which corresponds to the rotation frequency of the moving parts of the disperser $1500 \mathrm{rpm}$. The measurements were carried out at various temperatures of the model system in the temperature range from 30 to $80^{\circ} \mathrm{C}$.

Next, the experimental data were approximated by a polynomial function of the form:

$$
f(x)=a_{0}+\sum_{n} a_{n} \cdot x^{n}
$$

The dependences obtained for the corresponding model systems were plotted in the coordinates $\eta(\mathrm{Pa} \cdot \mathrm{s}) \times \mathrm{T}\left({ }^{\circ} \mathrm{C}\right)$.

According to the research results in [3], a group of authors chose the appropriate ratio of the mixture of gums for further research.

\section{Research results}

The main formulation components of all the model systems used for the developed fillings are xanthan gum and tara gum. Fig. 1 shows the dependence of the effective viscosity on the temperature of model systems, which contains xanthan gum and tare gum of various concentrations.



Fig. 1. Dependence of the effective viscosity on the temperature of the model system with xanthan gum and tara gum with different concentrations of the components:

$1-0.36 \%$ xanthan gum, $0.24 \%$ tara gum; $2-0.48 \%$ xanthan gum, $0.32 \%$ tara gum;

$$
3-0.6 \% \text { xanthan gum, } 0.4 \% \text { tara gum }
$$

The figure shows that an increase in the concentration of components in the model system is accompanied by an increase in its effective viscosity. This is obvious from the same dependences obtained separately for model systems with xanthan gum and tara gum, shown in Fig. 2, 3 respectively.

It can be seen from the given dependences that the dependence for a low concentration of the component in the model system, both for xanthan gum and tara gum, is the lowest relative to the 
axis on which the effective viscosity $\eta$ is plotted. According to the highest position relative to the $\mathrm{O} \times \eta$ axis is occupied by the dependence of the effective viscosity on the temperature of the model system with the highest of the investigated concentrations of these components.



Fig. 2. Dependence of the effective viscosity on the temperature of the model system with xanthan gum with concentration, $\%: 1-0.6 ; 2-0.8 ; 3-1$

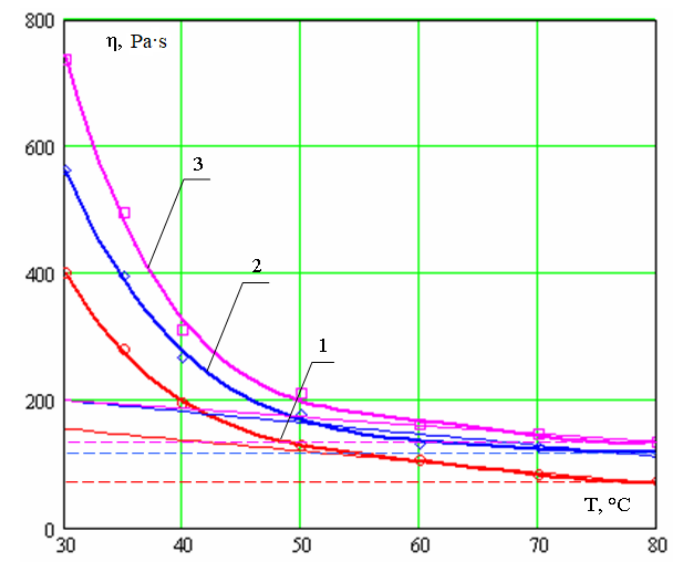

Fig. 3. Dependence of the effective viscosity on the temperature of the model system with tara gum with concentration, $\%: 1-0.6 ; 2-0.8 ; 3-1$

In this case, the dependences of the effective viscosity of model systems on their temperature in the temperature range from $30^{\circ} \mathrm{C}$ to $50^{\circ} \mathrm{C}$ are nonlinear. So for a decrease in the temperature of the mixture from $50{ }^{\circ} \mathrm{C}$ to a temperature of $40{ }^{\circ} \mathrm{C}$, the effective viscosity, for example, of a model system with a concentration of xanthan gum of $0.6 \%$, and a gum of tare $-0.4 \%$, increases 1.6 times. And for a temperature decrease from $40{ }^{\circ} \mathrm{C}$ to a temperature of $30{ }^{\circ} \mathrm{C}$, the effective viscosity increases 1.8 times. At the same time, for a decrease in temperature in the range from $60{ }^{\circ} \mathrm{C}$ to $50^{\circ} \mathrm{C}$ for the same sample, the effective viscosity increases only 1.4 times.

With an increase in temperature in the range from $50^{\circ} \mathrm{C}$ to $80^{\circ} \mathrm{C}$, the character of the given dependences approaches linear.

In order to identify the temperature of the model system, relative to which there is a sharp increase in the effective viscosity of the system, the experimental data were approximated by a linear function of the form:

$$
f(x)=b_{o}+b_{1} \cdot x
$$

The range of approximation data was chosen as follows. The first point of the data set, for which the linear approximation was carried out, on the temperature scale corresponded to 
the maximum temperature for which the experimental studies were carried out. So for the data from Fig. 1-3 it is equal to $80{ }^{\circ} \mathrm{C}$. The last point was chosen based on the correlation coefficient between the obtained linear approximation function (2) and the polynomial approximation function (1). The correlation coefficient was at least 0.95 .

In Fig. 1-3, the linear approximation function is shown by the solid line of the corresponding color. The dashed line indicates the minimum effective viscosity, which this model system has in the temperature range studied in the experiment.

Graphically, by the form of linear and polynomial approximation functions, it is possible to determine the temperature range in which a sharp increase in the effective viscosity of the model system begins.

Approximation functions for other model systems were determined using the same technique; they are shown in Fig. 4-7.

The presented dependences of the effective viscosity of the model systems from Fig. 4, 5-8 on their temperature have the same character as the dependence for a mixture of xanthan gum and tara gum. However, they have a number of features.

\section{Discussion of research results}

So for the model system containing xanthan gum $(0.6 \%)$, tara gum $(0.4 \%)$ and sugar with a concentration of $10,20,30$ and $40 \%$, the sample with a sugar concentration of $40 \%$ stands out. For an increase in sugar concentration by $10 \%$, it has a significantly higher effective viscosity in comparison with other samples. It is explained by a high sugar concentration with the possible formation of crystals due to the low amount of the liquid phase, that is, water. As a result, the model system acquires properties that are closer to an elastic-viscous body.

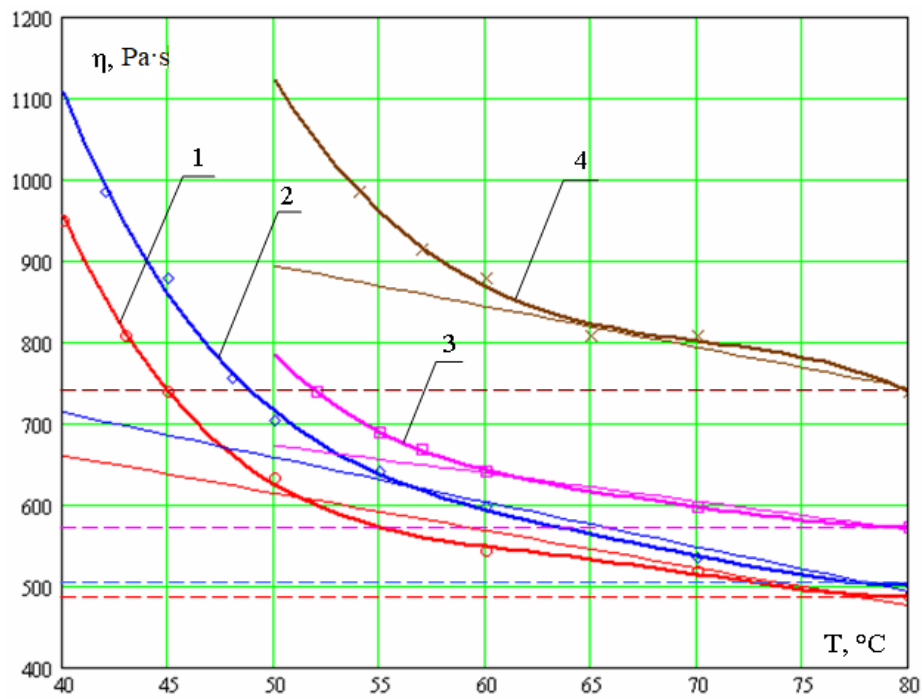

Fig. 4. Dependence of the effective viscosity on the temperature of the model system with xanthan gum $(0.6 \%)$, tara gum $(0.4 \%)$ and sugar with concentration, $\%$ :

$$
1-10 ; 2-20 ; 3-30 ; 4-40
$$

For a model system with xanthan gum $(0.6 \%)$, tara gum $(0.4 \%)$ and gelatin with a concentration of 1, 2, $3 \%$ (Fig. 5), the dependences of the effective viscosity on the temperature of a given system with different concentrations of gelatin differ from each other. other in the temperature range from $40{ }^{\circ} \mathrm{C}$ to $80{ }^{\circ} \mathrm{C}$ within no more than $5 \ldots 8 \%$. However, in the temperature range from $30{ }^{\circ} \mathrm{C}$ to $40{ }^{\circ} \mathrm{C}$, the dependences diverge, especially for the model system with a gelatin concentration of $3 \%$. As can be seen from Fig. 6, the dependence of the effective viscosity on the temperature of the sample with a gelatin concentration of $3 \%$ is higher relative to the axis on which the effective viscosity is plotted in comparison with other dependences. With an increase in the concentration of gelatin solutions, their viscosity increases nonlinearly along a curve that is in- 
versely convex to the concentration axis. One of the reasons for the sharp increase in viscosity with increasing concentration is the formation of structures in the system, that is, gelation.

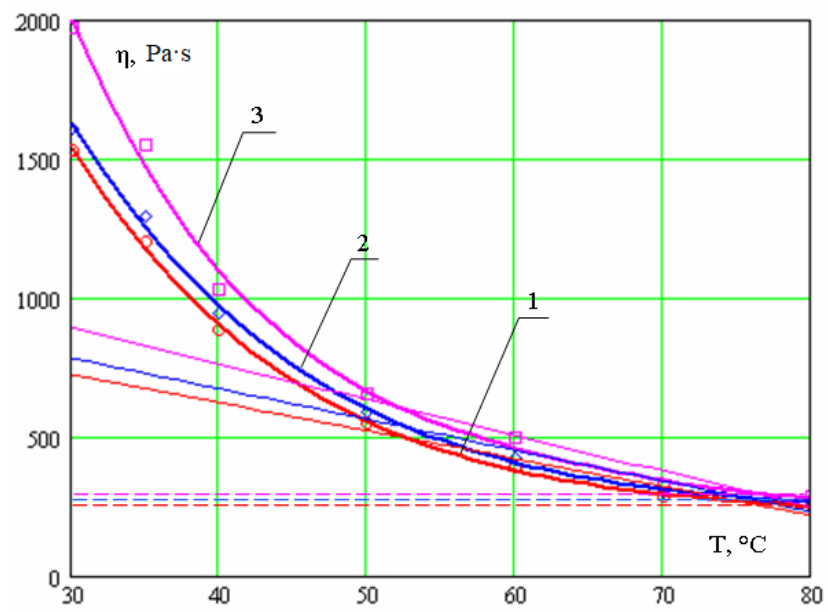

Fig. 5. Dependence of the effective viscosity on the temperature of the model system with xanthan gum $(0.6 \%)$, tara gum $(0.4 \%)$ and gelatin with concentration, $\%: 1-1 ; 2-2 ; 3-3$

Bonds are formed between the gelatin molecules, which lead to the formation of associates. An increase in the temperature of the system prevents gelation due to an increase in the intensity of Brownian motion and a decrease through it in the duration of the existence of bonds that arise between macromolecules. At the same time, a decrease in temperature promotes gelation, since this increases the number of contacts between macromolecules, which contributes to an increase in the strength of the so-called spatial network.

It is this property of gelatin solutions that is reflected in the nature of the dependence of the effective viscosity on the temperature of the model system with xanthan gum $(0.6 \%)$, tara gum $(0.4 \%)$ and gelatin. As a result of gelation in gelatin in the temperature range from $30{ }^{\circ} \mathrm{C}$ to $40{ }^{\circ} \mathrm{C}$, especially for a sample with a gelatin concentration of $3 \%$, an increase in effective viscosity occurs compared to other samples.

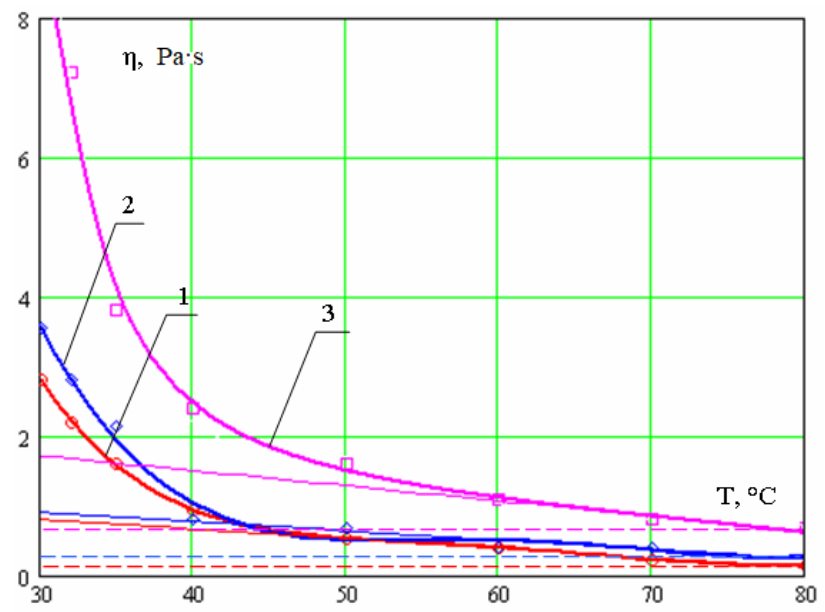

Fig. 6. Dependence of the effective viscosity on the temperature of the model system with gelatin with concentration, $\%: 1-1 ; 2-2 ; 3-3$

The dependences of the effective viscosity on the temperature of model systems with xanthan gum $(0.6 \%)$, tara gum $(0.4 \%)$ and skimmed milk powder with concentrations of 5,10 and $15 \%$ are shifted relative to the axis on which the effective viscosity is plotted in proportion to the concentration change dry skim milk. 
The viscosity of milk, as a rule, is influenced by emulsified and colloid-soluble particles, in particular the concentration of fat, the size of fat globules and their size distribution, the presence of agglomerates of fat globules. However, since the system contains skim milk, its addition reduces the amount of solvent in the system, corresponding to an increase in the concentration of the constituents in this model system. As a result, there is a corresponding change in the effective viscosity of the model system with xanthan gum, tara gum and skimmed milk powder.

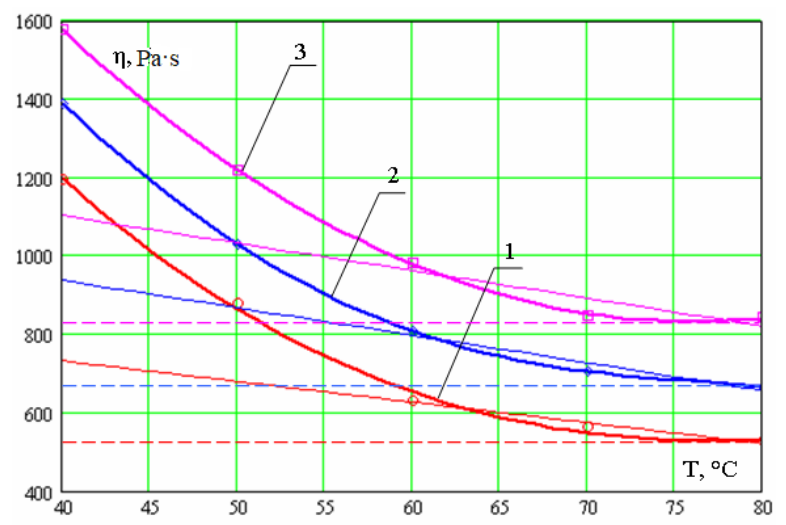

Fig. 7. Dependence of the effective viscosity on the temperature of the model system with xanthan gum $(0.6 \%)$, tara gum $(0.4 \%)$ and skimmed milk powder with a concentration, $\%$ : $1-5 ; 2-10 ; 3-15$



Fig. 8. Dependence of the effective viscosity on the temperature of the model system with xanthan gum $(0.6 \%)$, tara gum $(0.4 \%)$ and maltodextrin with concentration, $\%$ :

$$
1-2.5 ; 2-5 ; 3-7.5
$$

The dependences of the effective viscosity on the temperature of the model system with xanthan gum $(0.6 \%)$, tara gum $(0.4 \%)$ and maltodextrin with a concentration of $2.5,5$, and $7.5 \%$ differ from each other by no more than $3 . .6 \%$. This indicates that the addition of maltodextrin with a concentration of $2.5 \ldots 7.5 \%$ does not significantly affect the effective viscosity of the specified model system.

\section{Conclusions}

Dependences of the effective viscosity on the temperature of model systems with xanthan gum, tara gum, gelatin, sugar, skimmed milk powder and maltodextrin with different concentrations of these components were determined. It has been established that for the model system containing xanthan gum $(0.6 \%)$, tara gum $(0.4 \%)$ and sugar with a concentration of $10,20,30$ and $40 \%$, the sample with a sugar concentration of $40 \%$ is especially prominent. For an increase in sugar concentration by $10 \%$, it has a significantly higher effective viscosity in comparison with other samples. 
The dependence of the effective viscosity on the temperature of the model system with xanthan gum $(0.6 \%)$, tara gum $(0.4 \%$ ) and maltodextrin with a concentration of $2.5,5$, and $7.5 \%$ was found to differ from each other by no more than $3 . . .6 \%$. This indicates that the addition of maltodextrin with a concentration of $2.5 . .7 .5 \%$ does not significantly affect the effective viscosity of the specified model system.

The dependences of the effective viscosity on the temperature of model systems with xanthan gum $(0.6 \%)$, tara gum $(0.4 \%)$ and skimmed milk powder with concentrations of 5,10 and $15 \%$ are determined; they are displaced relative to the axis on which the effective viscosity is plotted proportional to the change in the concentration of skimmed milk powder.

The temperature range is established in which a sharp increase in the effective viscosity of the studied model systems begins, namely in the temperature range from $30^{\circ} \mathrm{C}$ to $40^{\circ} \mathrm{C}$, especially for a sample with a gelatin concentration of $3 \%$, an increase in the effective viscosity occurs in comparison with other samples.

The dependence of the effective viscosity of the model systems on their temperature in the temperature range from $30{ }^{\circ} \mathrm{C}$ to $50{ }^{\circ} \mathrm{C}$ has been found to be nonlinear. Thus, for a decrease in the temperature of the mixture from $50{ }^{\circ} \mathrm{C}$ to a temperature of $40{ }^{\circ} \mathrm{C}$, the effective viscosity of the model system with a concentration of xanthan gum of $0.6 \%$, and of a tara gum $-0.4 \%$, increases 1.6 times. And for a temperature decrease from $40{ }^{\circ} \mathrm{C}$ to a temperature of $30{ }^{\circ} \mathrm{C}$, the effective viscosity increases 1.8 times. At the same time, for a decrease in temperature in the range from $60{ }^{\circ} \mathrm{C}$ to $50{ }^{\circ} \mathrm{C}$ for the same sample, the effective viscosity increases only 1.4 times.

Thus, the dependences of the effective viscosity on the temperature of model systems with xanthan gum, tara gum, gelatin, sugar, skimmed milk powder and maltodextrin with different concentrations of these components are obtained. Based on the obtained dependences, the established temperature ranges in which a sharp increase in the effective viscosity of the studied model systems begins.

\section{References}

[1] Cherevach, E., Tenkovskaya, L. (2015). The development of technology of functional beverages based on whey and plant extracts. Food Processing: Techniques and Technology, 39 (4), 99-105.

[2] Rohart, A., Michon, C. (2014). Designing microstructure into xanthan gum-enriched acid milk gels. Innovative Food Science \& Emerging Technologies, 25, 53-57. doi: https://doi.org/10.1016/j.ifset.2014.01.002

[3] Pertsevoi, F., Bidyuk, D., Koshel, O. (2018). Analytical substantiation and choice of binary combination of polysaccharides for thermostenic milk-containing stuffing. Prohresyvni tekhnika ta tekhnolohiyi kharchovykh vyrobnytstv restorannoho hospodarstva i torhivli, 1 (27), 122-133.

[4] Iyrchenko, S. L., Kolesnikova, M. B. (2013). Creative prescription composition of fruit and berry fillings. Pratsi Tavriyskoho derzhavnoho ahrotekhnolohichnoho universytetu, 7 (13), 83-88.

[5] Tako, M. (1991). Synergistic interaction between xanthan and tara-bean gum. Carbohydrate Polymers, 16 (3), $239-252$. doi: https://doi.org/10.1016/0144-8617(91)90111-o

[6] Aymeson, A. (2012). Food thickeners, stabilizers, gel-forming agents. Sankt-Peterburg: «Professiya», 408.

[7] Phillips, G. O., Williams, P. A. (2006). Handbook of hydrocolloids. Sankt-Peterburg: GIORD, 536.

[8] Simsek, S. (2009). Application of xanthan gum for reducing syruping in refrigerated doughs. Food Hydrocolloids, 23 (8), 2354-2358. doi: https://doi.org/10.1016/j.foodhyd.2009.06.017

[9] Espert, M., Constantinescu, L., Sanz, T., Salvador, A. (2019). Effect of xanthan gum on palm oil in vitro digestion. Application in starch-based filling creams. Food Hydrocolloids, 86, 87-94. doi: https://doi.org/10.1016/j.foodhyd.2018.02.017

[10] Wu, Y., Ding, W., He, Q. (2018). The gelation properties of tara gum blended with $\kappa$-carrageenan or xanthan. Food Hydrocolloids, 77, 764-771. doi: https://doi.org/10.1016/j.foodhyd.2017.11.018 